\title{
Incremental Loop Closure Verification by Guided Sampling
}

\author{
Tanaka Kanji
}

\begin{abstract}
Loop closure detection, the task of identifying locations revisited by a robot in a sequence of odometry and perceptual observations, is typically formulated as a combination of two subtasks: (1) bag-of-words image retrieval and (2) post-verification using RANSAC geometric verification. The main contribution of this study is the proposal of a novel post-verification framework that achieves good precision recall trade-off in loop closure detection. This study is motivated by the fact that not all loop closure hypotheses are equally plausible (e.g., owing to mutual consistency between loop closure constraints) and that if we have evidence that one hypothesis is more plausible than the others, then it should be verified more frequently. We demonstrate that the problem of loop closure detection can be viewed as an instance of a multi-model hypothesize-and-verify framework and build guided sampling strategies on the framework where loop closures proposed using image retrieval are verified in a planned order (rather than in a conventional uniform order) to operate in a constant time. Experimental results using a stereo SLAM system confirm that the proposed strategy, the use of loop closure constraints and robot trajectory hypotheses as a guide, achieves promising results despite the fact that there exists a significant number of false positive constraints and hypotheses.
\end{abstract}

\section{INTRODUCTION}

Loop closure detection, the task of identifying locations revisited by a robot in a sequence of odometry and perceptual observations, is an important first step to estimate robot trajectory and has contributed to important applications including localization \& mapping [1], landmark discovery [2], image alignment [3], topological mapping [4], and place recognition [5]. Failure in loop closure detection can yield catastrophic damage in an estimated robot trajectory, and achieving an acceptable tradeoff between precision and recall is critical in this context. In previous research [5][7], loop closure detection is typically formulated as a bagof-words image retrieval problem where a query/database image is represented by an unordered collection of vector quantized local invariant features termed visual words and then efficiently indexed and retrieved to identify pairs of matched locations using each view image as a query input. However, even state-of-the-art image retrieval techniques generate a considerable number of false positives owing to confusing features and perceptual aliasing [5]. Therefore, the image retrieval process is typically followed by a postverification step using robust RANSAC verification methods [8]. However, the post-verification step is a computationally intensive process, which requires quadratic time in the length of a view sequence. Therefore, improving the total cost

Our work has been supported in part by JSPS KAKENHI Grant-in-Aid for Young Scientists (B) 23700229, and for Scientific Research (C) 26330297.

K. Tanaka is with Graduate School of Engineering, University of Fukui, Japan. tnkknj@u-fukui.ac.jp for performance of loop closure detection is an important practical problem and main focus of this study.

In this study, we address the above issue with guided sampling. Unlike previous frameworks where latest loop closure constraints $z_{t}$ (proposed by image retrieval) are verified individually in a uniform order at each location $t$, in the proposed framework, a constant number of constraints are intelligently sampled in a planned order from all the $O\left(t^{2}\right)$ constraints obtained to date. This study is motivated
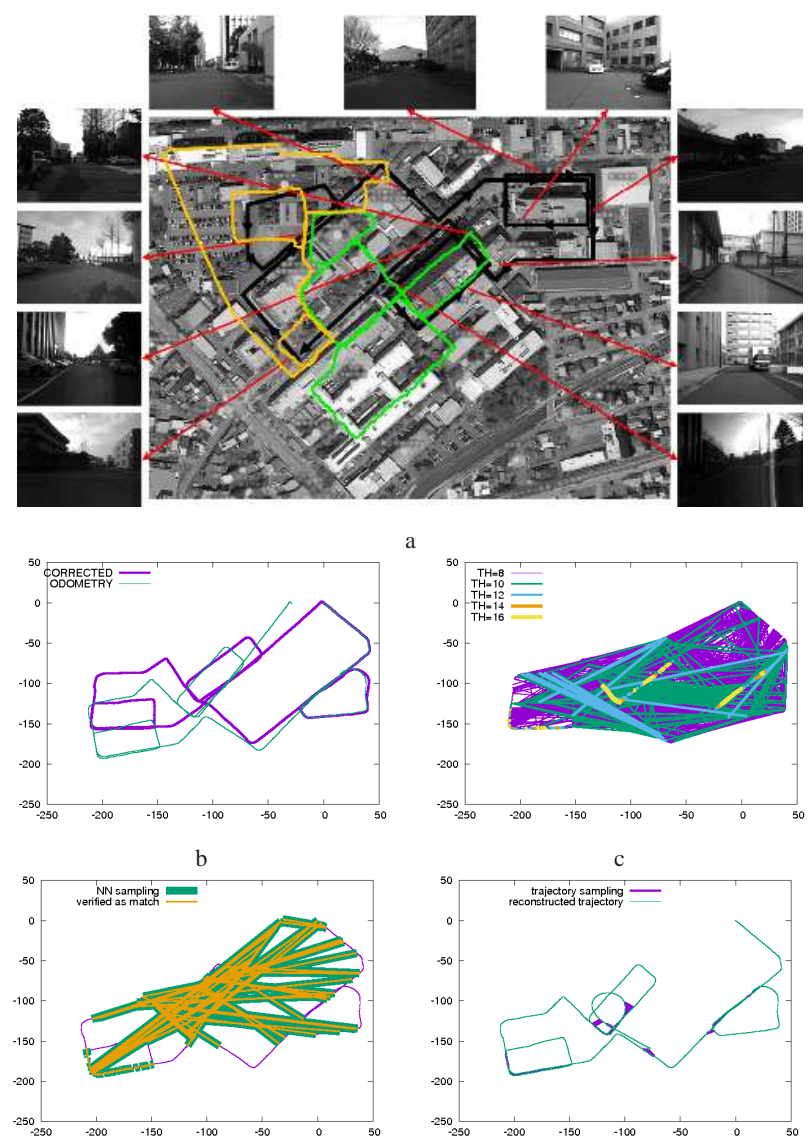

e

Fig. 1. Guided sampling for loop closure verification. Presented in (a) is our experimental environment with robot trajectory \#1 (black), \#2 (green), and \#3 (orange). Loop closure detection is an essential task for correcting the accumulated error in visual odometry (b). Indicated in (c) are typical results of detecting loop closure constraints by FAB-MAP image retrieval + RANSAC post-verification, for different settings of RANSAC thresholds, $\mathrm{TH}=8,10,12,14,16$. One can see that the detection performance in terms of precision and recall is significantly less than perfect and that high recall is achieved when $\mathrm{TH}=8$ or 10 at the cost of extremely low precision. The basic idea of guided sampling is to verify loop closure hypotheses in a planned order (rather than in conventional uniform order) by exploiting a domain specific knowledge of mutual consistency between loop closure constraints. Illustrated in (d) and (e) are sample results of guided sampling using two different strategies, called NN sampling and trajectory sampling. 
by the fact that not all loop closure hypotheses are equally plausible (e.g., owing to mutual consistency between loop closure constraints [9]) and that if we have evidence that one hypothesis is more plausible than the others, then it should be verified more frequently. Examples of such plausible hypotheses include:

1) a loop closure constraint that provides a plausible reconstruction of a robot trajectory;

2) a loop closure constraint that is consistent with a plausible robot trajectory hypothesis;

3) a loop closure constraint that is spatially similar to a plausible loop closure constraint.

To implement the above idea, we cast loop closure detection as an instance of a multi-model hypothesize-and-verify problem where a set of hypotheses of robot trajectory is hypothesized from loop closure constraints and verified in terms of consistency against other loop closure constraints. The proposed approach is motivated by three independent observations. First, we are inspired by the recent success of guided sampling strategies in hypothesize-and-verify techniques (e.g., USAC [10]). Second, loop closure detection is essentially a multi-model estimation problem [11], rather than the single model estimation considered in classical applications of the hypothesize-and-verify approach (e.g., structure-from-motion [10]), where the goal is to identify multiple instances of models (i.e., loop closure hypotheses) and the inliers to one model behave as pseudo-outliers to the other models. Finally, and most importantly, the framework is sufficiently general and effective for implementing various guided sampling strategies that implement the domain knowledge presented above.

Although the proposed approach is general, we focus on a challenging scenario of stereo SLAM, which has been attracting increasing interest in recent years [12], to demonstrate the efficacy of the proposed system. Our experiments employ a stereo SLAM system that implements odometry using stereo visual odometry as in [13], loop closure detection using appearance-based image retrieval with SURF local features and bag-of-words image model as in [5], postverification using RANSAC geometric verification on local feature keypoints as in [8], and pose graph SLAM as in [14]. Fig 1b,c illustrate an odometry-based robot trajectory, trajectory corrected by loop closing, and a set of loop closure constraints determined by employing the two strategies. As can be observed, significant errors in trajectory are accumulated as the robot navigates and the errors are successfully corrected given correct loop closure constraints. It can also be seen that image retrieval-based loop closure detection and RANSAC post-verification are both less than perfect (Fig. 11); there are a significant number of false positives and negatives. These two types of errors, accumulated errors in odometry and misrecognition in detection and postverification of errors, are the main error sources that we address in this study. Experimental results using our stereo SLAM system confirm that the proposed strategy, the use of loop closure constraints and robot trajectory hypotheses as a guide, achieves promising results despite the fact that there are a significant number of false positive constraints and hypotheses (Fig. 2).

\section{A. Related Work}

This study can be viewed as a novel application of multimodel-based consensus approaches (e.g., multi-RANSAC [11]). Similar to previous multi-model approaches, we focus on determining multiple plausible hypotheses (i.e., loop closure hypotheses) rather than the single best hypothesis.

We allow a set of detected models (i.e., loop closure constraints) to be partially inconsistent with each other. Such partial inconsistency in loop closure constraints can be resolved reliably by employing modern SLAM back-ends such as robust pose graph optimization in [9].

Most of the existing works on loop closure detection have focused on the image retrieval step in the task, rather than the post-verification step [15]. In fact, loop closure detection techniques are typically classified in terms of image retrieval strategies (rather than post-verification strategies) [16]. Images are typically represented by a collection of invariant local descriptors [5] or a global holistic descriptor [7], [17]. Loop closure detection has been employed by many SLAM systems [18]-[21]. However, the above works did not focus on the post-verification step or introduce novel insight to the guided sampling strategy.

Guided sampling has been studied in many matching problems that are closely related to loop closure detection with respect to its objective. In [22], techniques were presented for improving the speed of robust motion estimation based on the guided sampling of image features, which is inspired by the MLESAC algorithm. [23] presented an approach called double-window optimization where a place recognition is used to identify loop-closing constraints and incorporate the constraints into the optimization. [24] explored an approach based on considering features in the scene database and matching them to query image features as opposed to previous methods that match image features to visual words or database features, and presented an efficient solution based on prioritized feature matching. [25] proposed a method for improved geometric verification by exploiting the statistics of image collections and gathering information during geometric verification, to improve the overall efficiency. [26] presented an unsupervised learning approach for learning threshold for geometric verification. Recently, a series of robust SLAM back-end algorithms that allow misrecognition in loop closure constraints have been studied [9]. However, their objectives are neither the guided sampling nor SLAM front-end applications; rather, they follow conventional batch-style matching. Moreover, differing from general purpose matching algorithms, we are interested in and focus on the use of task specific knowledge regarding loop closure detection to improve overall performance.

This paper is a part of our studies on loop closure detection. Recently, we have discussed cross-season place recognition [27], part-based scene modeling [28], landmark discovery [29], and map descriptor [30] in IROS15, ICRA15, 
PPNIV15 papers. Guided sampling in loop closure detection has not been addressed in the above papers.

\section{APPROACH}

\section{A. Loop Closure Detection}

For clarity of presentation, we first describe a baseline SLAM system where the proposed approach is built and used as a benchmark for performance comparison in the experimental section. As mentioned, we build the proposed system on a stereo SLAM system where a stereo vision sensor is employed for both visual odometry [13] and visual feature acquisition [5] and follow the standard formulation of pose graph SLAM [31]. In pose graph SLAM, the robot is assumed to move in an unknown environment, along a trajectory described by a sequence of random variables $x_{1: T}=x_{1}, \cdots, x_{T}$. While moving, it acquires sequences of odometry measurements $u_{1: T}=u_{1}, \cdots, u_{T}$ and perception measurements $z_{1: T}=z_{1}, \cdots, z_{T}$. Each odometry measurement $u_{t}(1 \leq t \leq T)$ is a pairing of rotation and translation acquired by visual odometry. Each perceptual measurement $z_{t}$ is a set of loop closure constraints $z_{t}^{1}, \cdots, z_{t}^{N_{t}}$, each of which is a pair of location IDs, $t, t^{\prime}$ with a likelihood score that represents the likelihood of the location pair belonging to the same place, which is acquired by FAB-MAP in our case. More formally, we begin with an empty list of loop closure constraints. At each time $t$, we execute FAB-MAP using the latest visual image as a query to identify the top $N_{t}=50$ ranked images that receive the highest likelihood scores. We then insert the $N_{t}=50$ pairs from the query image and each of the $N_{t}$ top-ranked database images as new constraints to the list.

For simplicity, we begin by assuming that fixed sets of loop closure constraints $z_{1: T}$ and map hypotheses $m_{1: M}$ are a priori given; typical hypothesize-and-verify algorithms require such a fixed set assumption [11]. Clearly, this assumption is violated in our SLAM applications as both the loop closure constraints and the map hypotheses must be incrementally derived as the robot navigates. This incremental setting will be addressed in Section $\amalg-C$ by relaxing the fixed set assumption. We divide the entire measurement sequence into constant time windows and generate one hypothesis per window. To generate a hypothesis, we employ pose graph SLAM that expects the following as input: (1) the single loop closure constraint whose score received from FABMAP is the highest within the time window of interest, and (2) a sequence of previous odometry measurements. This yields $M=T / W$ map hypotheses when the size of the time window is $W$. In experiments, we set the time window size sufficiently small, $W=10$, that appearance of images do not change significantly within time window.

Performance of loop closure detection is typically evaluated by precision-recall. This performance measure requires a set of ground truth loop closure constraints and a set of constraints verified as matched by the RANSAC. We run the RANSAC for each loop closure constraint (selected by the guided sampling) that consists of a pair of images to check if the keypoint configuration is geometrically consistent between the image pair. We use RANSAC verification with the fundamental matrix in [8] and a preset threshold of " 8 ", as indicated in Fig 1c. If the RANSAC score exceeds the threshold, the input pair is verified as matched. For each query image $i$, we define a range of ground-truth loop closure constraints in the form: $\left(i, j_{i}^{\text {begin }}\right), \cdots,\left(i, j_{i}^{\text {end }}\right)$, and consider a verified constraint $(i, j)$ is correct if and only if $j \in\left[j_{i}^{\text {begin }}, j_{i}^{\text {end }}\right]$.

Based on the above terminology, we formulate the problem of guided sampling in loop closure detection. Let $s$ denote a selection of a loop closure constraint. Recalling that a set of $N_{t}$ new constraints arrive at each time instance $t$, we represent a selection $s$ of loop closure constraints by:

$$
s=(t, n) \quad t \in[1, T], n \in\left[1, N_{t}\right] .
$$

Let $v_{i}$ denote the result of post-verification:

$$
v_{i}=V\left(s_{i}\right) \quad v_{i} \in\{0,1\},
$$

which indicates if the RANSAC score exceeds a predefined threshold ("1") or not ("0"). Guided sampling is the problem of selecting the next constraint to verify:

$$
s_{i}=S\left(u_{1: t}, z_{1: t}, s_{1: i-1}, v_{1: i-1}\right),
$$

given a history of previous odometry $u_{1: t}$ and perception $z_{1: t}$, and a history of previous selections $s_{1: i-1}$, and verification results $v_{1: i-1}$.

\section{B. Guided Sampling Strategies}

A naive strategy for guided sampling is to uniformly sample one constraint/hypothesis from a history of previously acquired constraints/hypotheses. This strategy is straightforward and easy to implement. Unfortunately, it does not achieve acceptable precision-recall tradeoff as indicated in the experimental section (Section III). To achieve improved precision-recall tradeoff, we present several different strategies for guided sampling in the following discussion.

The first strategy, termed trajectory sampling (TS), is a strategy that samples loop closure constraints that are consistent with a robot trajectory hypothesis (Fig. 1 ). The basic idea is to verify the consistency between a pair of constraints using the robot trajectory hypothesis as an intermediate. This strategy selects one random robot trajectory hypothesis $h$ and then samples a loop closure constraint, a pair of IDs $\left(t, t^{\prime}\right)$ of locations that are near each other:

$$
\left\|p(t, h)-p\left(t^{\prime}, h\right)\right\|<T_{p}
$$

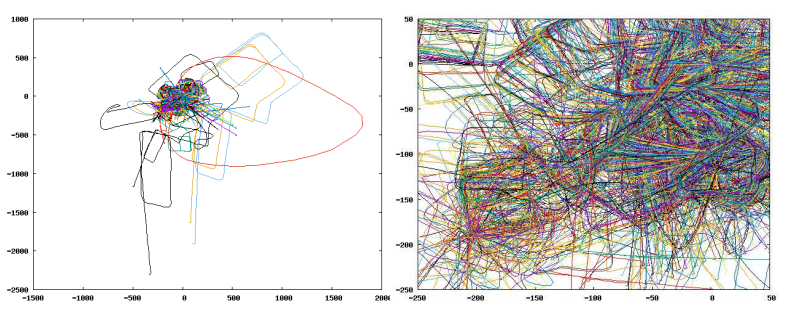

Fig. 2. All of the 538 robot trajectory hypotheses generated in Experiment $\# 1$. On the right is a close-up of the figure on the left. 
where $p(t, h)$ is the 2-dimensional coordinate of location $t$ conditioned on a robot trajectory hypothesis $h$, and $T_{p}$ is a preset threshold, $10 \mathrm{~m}$.

The second strategy, termed neighbor sampling (NS), is a strategy that samples loop closure constraints that are neighbors to plausible constraints. Fig. 1 presents examples of neighbor sampling. This strategy is motivated by the fact that in street-like environments, there often exists a sequence of matched views, rather than single isolated matches. This strategy selects one random trajectory hypothesis and one random verified loop closure constraint $(i, j)$ on the selected trajectory, and then samples one of its four neighbors $(i \pm$ $1, j \pm 1)$.

The third strategy, termed breadth first (BF), is a strategy that samples trajectory hypotheses in a breadth-first order, rather than in a uniform order. This strategy is motivated by a limitation of uniform samplings in an incremental scenario, that is, the total number of old sampling hypotheses generated at the beginning of a robot's navigation tends to be considerably greater than the new hypotheses generated at the end of the navigation. As a result, the distribution of "being sampled" becomes considerably unbalanced among the hypotheses as indicated in Fig. 4b "UNIFORM SAMPLING". To address this limitation, this strategy continuously monitors the number of individual hypotheses being sampled to date and selects the hypothesis with the least number of being sampled as the next hypothesis to sample.

The fourth strategy, termed depth first (DF), is a strategy that samples a trajectory hypothesis according to the importance weight, which is evaluated by the number of loop closure constraints that are consistent with the hypothesis of interest and have been verified as matched to date. Currently, we divide the hypothesis set into two subsets, upper half and lower half, according to the importance weight, and then sample the next hypothesis from the former subset.

We need to select one guided sampling strategy at a time among the several different strategies for the sampling constraints (TS, NS) and hypotheses (BF, WS) discussed above. Currently, we introduce a random selection rule using preset parameters $P_{T S}, P_{N S}, P_{B F}$ and $P_{D F}$. We randomly select one of the three different strategies for hypothesis sampling, BF, DF, and uniform strategies with probabilities $P_{B F}: P_{D F}:\left(1-P_{D F}-P_{B F}\right)$. We also randomly select one of the three different strategies for constraint sampling, TS, NS, and uniform strategies with probabilities $P_{T S}: P_{N S}$ : $\left(1-P_{T S}-P_{N S}\right)$. In experiments, we test the different settings of these probabilities to investigate the contributions of the different sampling strategies.

\section{Incremental Extension}

In this section, we relax the fixed set assumption in Section II-A and consider the general incremental setting of loop closure detection. As a main extension, the system must update the set of loop closure constraints and set of map hypotheses. Further, because sampling strategies introduced in Section $\amalg-A$ rely on the knowledge of mutual consistency between loop closure constraints and map hypotheses, it also must update this knowledge every time a new constraint or hypothesis arrives. Thus, we introduce the concept of consistency matrix: $C$, which is an $N_{t} T \times M$ sized matrix defined as consistency $C_{i j}$ between each $i$-th loop closure constraint and $j$-th map hypothesis, and we incrementally update it every time a new constraint or hypothesis arrives. The update for a new constraint and hypothesis requires $M$ and $N_{t} T$ computations of consistency, respectively. In the moderate-sized environments (e.g., $M \leq 1000, N_{t} T \leq$ $1 \times 10^{5}$ ) considered in the experimental section, we can assume these two costs are negligible compared to the costs for loop closure verification and map reconstruction.

\section{EXPERIMENTS}

\section{A. Settings}

We conducted loop closure detection experiments using a stereo SLAM system on a university campus. Our experiments employed a stereo SLAM system that implemented the proposed guided sampling strategies. The principal steps involved visual odometry, loop closure detection, and postverification. The first step executed stereo visual odometry to reconstruct the robot trajectory. We adopted the stereo visual odometry algorithm proposed in [13], which has proven to be effective in recent visual odometry applications (e.g., [32]). The second step applied the appearance-based image
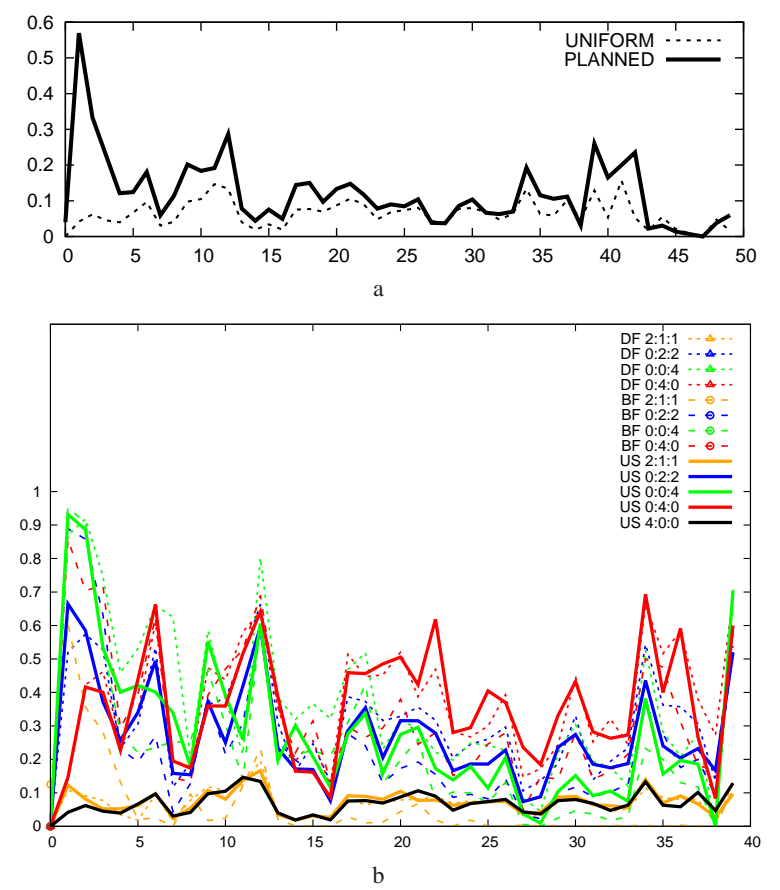

Fig. 3. Effectiveness of using robot trajectory hypotheses as a guide for sampling loop closure constraints. (a) is a histogram of the success ratio of detecting correct loop closure hypotheses for two distinct cases: sampling is guided ("PLANNED") and not guided ("UNIFORM"). The horizontal axis represents the accuracy of the robot trajectory hypotheses, in terms of error $[\mathrm{m}]$ in robot location on the reconstructed trajectory with respect to the ground-truth trajectory. (b) compares success ratio for different combinations of guided sampling strategies. In the legend, "DF", "BF", and "US" are the depth-first, breadth-first, and uniform sampling strategies, respectively, in Section $\amalg-\mathrm{B}$ "x:y:z" indicates that the parameters $P_{T S}, P_{N S}$ for switching strategies are set to $P_{T S}=z /(x+y+z)$ and $P_{N S}=y /(x+y+z)$. 

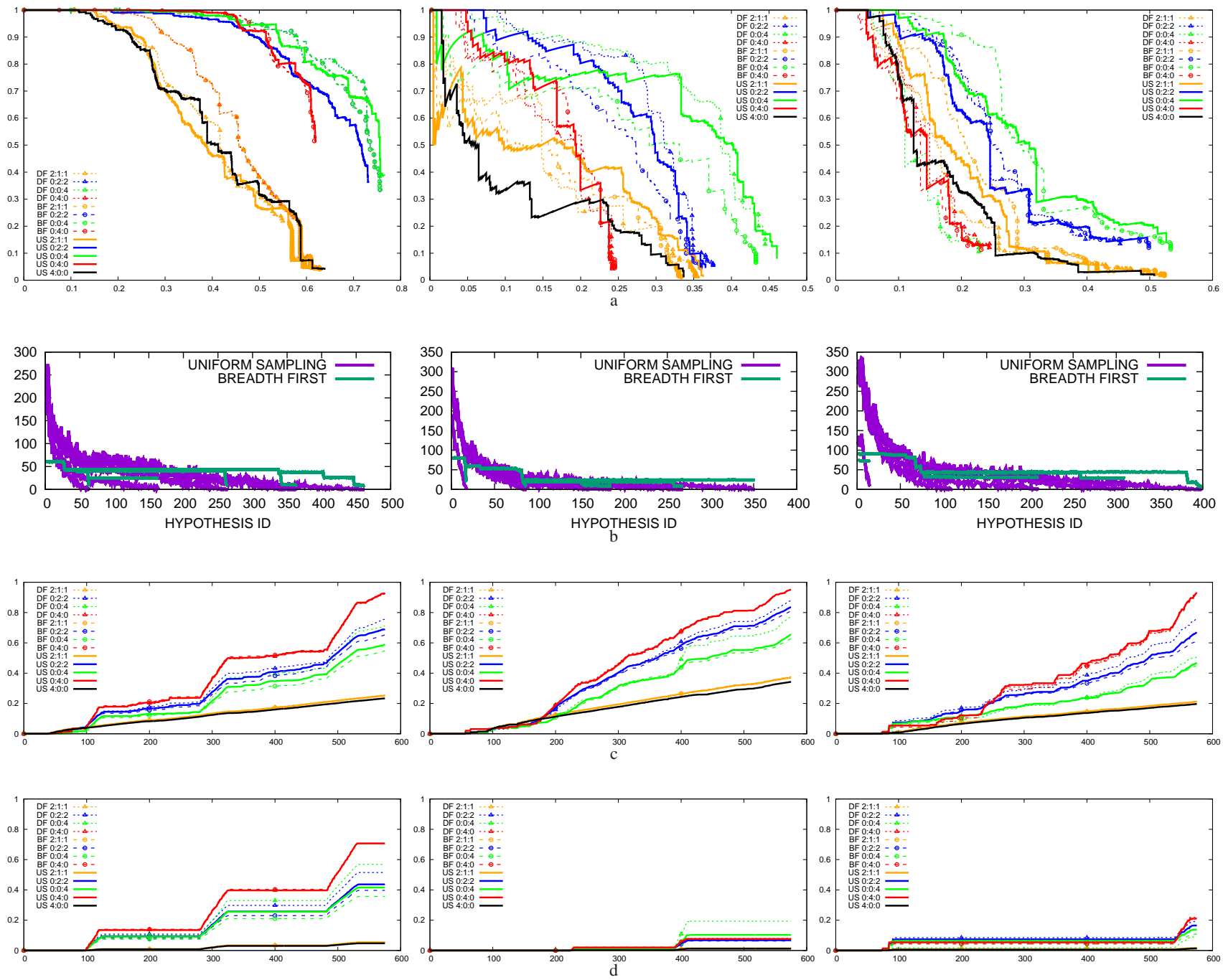

Fig. 4. Performance results. From left to right, the results for three different experiments, \#1, \#2, and \#3, are presented. From top to bottom, each panel presents (a) the precision-recall curve, (b) a comparison of total number of individual hypotheses being sampled between uniform sampling and breadth first strategies, (c) ratio of loop closure constraints that are guided by individual strategies and also verified as matched by RANSAC (horizontal axis: time window ID), and (d) ratio of loop closure constraints that are guided by individual strategies, verified as matched by RANSAC, and are correct constraints with respect to the ground-truth (horizontal axis: time window ID).

retrieval, FAB-MAP, proposed in [5]. This step generated a set of $N_{t}$ new loop closure constraints and inserted these into the constraint list as indicated in Section $\amalg-\mathrm{A}$. The third step performed guided sampling to select a set of loop closure constraints to verify and applied each of these to RANSAC verification to be classified as matched or unmatched.

Fig. 11,b present the ground-truth robot trajectories and visual odometry trajectories superimposed on Google map imagery. The ground-truth trajectories were generated using a SLAM algorithm based on the graph optimization in [14] using manually identified ground-truth loop closure constraints as input. As indicated, significant odometry errors were accumulated as the robot navigated. We collected three sequences along routes with travel distances of $1364 \mathrm{~m}, 1020$ $\mathrm{m}$, and $1250 \mathrm{~m}$, using a cart equipped with a Bumblebee stereo vision camera system, as illustrated in Fig 1h. We defined a ground-truth loop closure constraint as a pairing of two locations $i, j$ whose distance was less than $10 \mathrm{~m}$. Occlusion was severe in the scenes and people and vehicles were dynamic entities occupying the scenes. We processed each path and collected stereo image sequences with lengths 5759, 6358, and 6034 .

\section{B. Proof-of-Concept Experiment}

In this paper, we rely on an assumption that robot trajectory hypotheses act as a guide to sample a new good loop closure constraint. One could argue that because an excessive number of false positive hypotheses exist, as indicated in Fig. 2. that the guide could cause more harm than assistance. As a proof-of-concept experiment, we experimentally compared the ratio of successful loop closure detection for three distinct cases: (1) not guided, (2) guided by a correct robot trajectory 

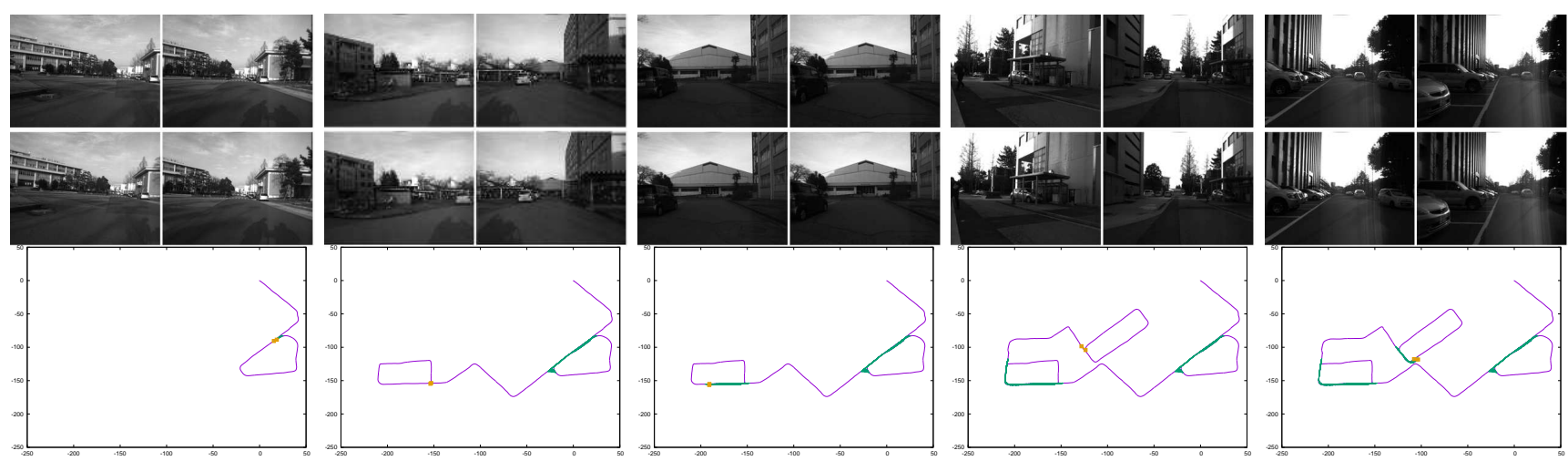

neighbor sampling
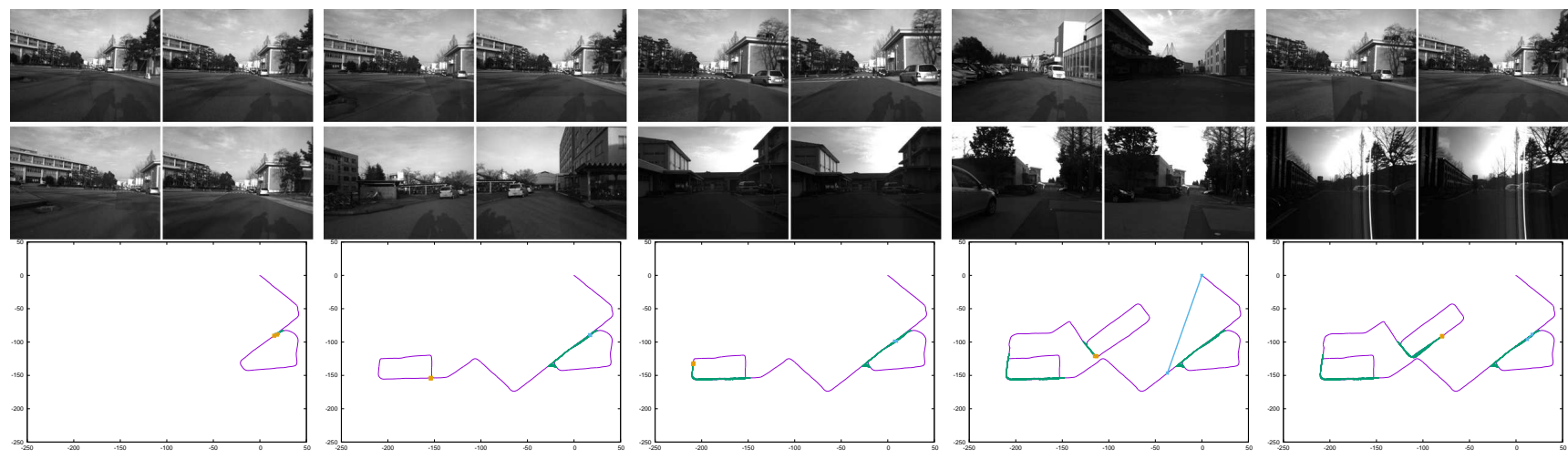

trajectory sampling

Fig. 5. Examples of correct loop closure constraints proposed by two different guided sampling strategies. For each example, the top and bottom panel represent two different loop closure constraints $s^{t o p}=(i, j)$ and $s^{b o t t o m}=\left(i^{\prime}, j^{\prime}\right)$ using the corresponding image pairs $(i, j)$ and $\left(i^{\prime}, j^{\prime}\right)$, where the former constraint $s^{\text {top }}$ is used as a guide to sample the latter (and correct) constraint $s^{\text {bottom }}$.

hypothesis, and (3) guided by an incorrect robot trajectory hypothesis. To evaluate (2) and (3), we collected sets of robot trajectory hypotheses with different levels of correctness, which were measured in terms of percentage of correct loop closure constraints hypothesized by the hypothesis of interest. Fig. 3 presents the success ratio for loop closure detection guided by robot trajectory hypotheses. It can be seen that the proposed guided sampling does not harm the process, even when an inaccurate (false positive) hypothesis is used and that it does help when an accurate hypothesis is used. We can observe that sampling guided by false positive hypotheses frequently behaves similarly to uniform sampling, as the robot's poses on false positive hypotheses typically distribute uniformly over the environment, as indicated in Fig. 2 .

\section{Precision-Recall Performance}

We evaluated the proposed strategies for guided sampling. We employed precision and recall as introduced in Section II-A as the performance measure. We did not use trivial loop closure constraints where the travel distance between the image pairs was overly short, less than $100 \mathrm{~m}$. That is, loop closures were not detected until the robot had travelled $100 \mathrm{~m}$ from the start location in all experiments.

Fig 4 th presents the precision-recall curves. It can be observed that the TS strategy method outperformed all the other strategies considered in the current experiments. We observe that TS strategy was able to use robot trajectory hypotheses as an effective guide to propose good loop closure constraint, despite the fact that majority of robot trajectories are partially incorrect with respect to the ground truth as shown in Fig. 2.

\section{Examples of Guided Sampling}

Fig. 5 presents examples of correct loop closure constraints proposed by guided sampling. We tested two different strategies: neighbor sampling, which uses a previously verified loop closure constraint as a guide and samples its neighbor constraints, and trajectory sampling, which uses robot trajectories reconstructed from previously verified loop closure constraints as a guide and samples constraints that are consistent with a reconstructed trajectory. It can be seen that the neighbor sampling strategy contributed to detecting loop closure constraints of similar scenes to previously detected loop closure constraints; whereas, the trajectory sampling strategy tended to detect loop closure constraints of extremely dissimilar scenes.

\section{E. Ratio of Verified/Matching Constraints}

Fig. 4 and Fig. 4 $\mathrm{d}$ present the ratio of loop closure constraints that are verified as matched and are correct with respect to the ground-truth. It can be seen that the neighbor sampling strategy outperformed the other strategies considered in finding matched and correct constraints. We 
observe that neighbor sampling strategy was effective to sample good constraints especially when almost all the robot trajectories reconstructed to date are incorrect with respect to the ground-truth.

\section{CONCLUSIONS}

The main contribution of this study is the proposal of a novel post-verification framework that achieves good precision recall trade-off in loop closure detection. We showed the loop closure detection can be viewed as an instance of multimodel hypothesize-and-verify framework, and based on an incremental extension of this framework, we built strategies for guided sampling, by which loop closures proposed by image retrieval are verified in a planned order rather than in a conventional uniform order to operate in a constant time. Experimental results using a stereo SLAM system confirmed that the proposed strategy, the use of loop closure constraints and robot trajectory hypotheses as a guide, achieves promising results despite the fact that there exists a significant number of false positive constraints and hypotheses.

\section{REFERENCES}

[1] D. Hähnel, W. Burgard, D. Fox, and S. Thrun, "An efficient fastslam algorithm for generating maps of large-scale cyclic environments from raw laser range measurements," in 2003 IEEE/RSJ International Conference on Intelligent Robots and Systems, Las Vegas, Nevada, USA, October 27 - November 1, 2003, 2003, pp. 206-211.

[2] H.-K. Tan and C.-W. Ngo, "Common pattern discovery using earth mover s distance and local flow maximization," in IEEE Int. Conf. Computer Vision (ICCV), 2005, pp. 1222-1229.

[3] T. Zhou, Y. Jae Lee, S. X. Yu, and A. A. Efros, "Flowweb: Joint image set alignment by weaving consistent, pixel-wise correspondences," in Proceedings of the IEEE Conference on Computer Vision and Pattern Recognition, 2015, pp. 1191-1200.

[4] G. Blanc, Y. Mezouar, and P. Martinet, "Indoor navigation of a wheeled mobile robot along visual routes," in Robotics and Automation, 2005. ICRA 2005. Proceedings of the 2005 IEEE International Conference on. IEEE, 2005, pp. 3354-3359.

[5] M. Cummins and P. M. Newman, "Appearance-only SLAM at large scale with FAB-MAP 2.0," I. J. Robotic Res., vol. 30, no. 9, pp. 1100-1123, 2011. [Online]. Available: http://dx.doi.org/10.1177/0278364910385483

[6] K. Pirker, M. Rüther, and H. Bischof, "CD SLAM continuous localization and mapping in a dynamic world," in 2011 IEEE/RSJ International Conference on Intelligent Robots and Systems, IROS 2011, San Francisco, CA, USA, September 25-30, 2011, 2011, pp. 3990-3997. [Online]. Available: http://dx.doi.org/10.1109/IROS.2011.6094588

[7] D. Gálvez-López and J. D. Tardós, "Bags of binary words for fast place recognition in image sequences," IEEE Transactions on Robotics, vol. 28, no. 5, pp. 1188-1197, 2012. [Online]. Available: http://dx.doi.org/10.1109/TRO.2012.2197158

[8] K. Lebeda, J. Matas, and O. Chum, "Fixing the locally optimized RANSAC," in British Machine Vision Conference, BMVC 2012, Surrey, UK, September 3-7, 2012, 2012, pp. 1-11. [Online]. Available: http://dx.doi.org/10.5244/C.26.95

[9] N. Sunderhauf and P. Protzel, "Switchable constraints vs. max-mixture models vs. rrr-a comparison of three approaches to robust pose graph slam," in Robotics and Automation (ICRA), 2013 IEEE International Conference on. IEEE, 2013, pp. 5198-5203.

[10] R. Raguram, O. Chum, M. Pollefeys, J. Matas, and J. Frahm, "Usac: a universal framework for random sample consensus," Pattern Analysis and Machine Intelligence, IEEE Transactions on, vol. 35, no. 8, pp. 2022-2038, 2013.

[11] Y. Kanazawa and H. Kawakami, "Detection of planar regions with uncalibrated stereo using distributions of feature points." in BMVC. Citeseer, 2004, pp. 1-10.

[12] C. Beall and F. Dellaert, "Appearance-based localization across seasons in a metric map," in IROS14 WS PPNIV, 2014.
[13] A. Geiger, J. Ziegler, and C. Stiller, "Stereoscan: Dense 3d reconstruction in real-time," in Intelligent Vehicles Symposium (IV), 2011 IEEE. IEEE, 2011, pp. 963-968.

[14] M. Kaess, A. Ranganathan, and F. Dellaert, "isam: Incremental smoothing and mapping," Robotics, IEEE Transactions on, vol. 24, no. 6, pp. 1365-1378, 2008.

[15] B. P. Williams, M. Cummins, J. Neira, P. M. Newman, I. D. Reid, and J. D. Tardós, "A comparison of loop closing techniques in monocular SLAM," Robotics and Autonomous Systems, vol. 57, no. 12 , pp. 1188-1197, 2009. [Online]. Available: http://dx.doi.org/10.1016/j.robot.2009.06.010

[16] —, "An image-to-map loop closing method for monocular SLAM," in 2008 IEEE/RSJ International Conference on Intelligent Robots and Systems, September 22-26, 2008, Acropolis Convention Center, Nice, France, 2008, pp. 2053-2059. [Online]. Available: http://dx.doi.org/10.1109/IROS.2008.4650996

[17] G. Klein and D. W. Murray, "Improving the agility of keyframebased SLAM," in Computer Vision - ECCV 2008, 10th European Conference on Computer Vision, Marseille, France, October 12-18, 2008, Proceedings, Part II, 2008, pp. 802-815. [Online]. Available: http://dx.doi.org/10.1007/978-3-540-88688-4_59

[18] J. Civera, O. G. Grasa, A. J. Davison, and J. Montiel, "1-point ransac for ekf-based structure from motion," in Intelligent Robots and Systems, 2009. IROS 2009. IEEE/RSJ International Conference on. IEEE, 2009, pp. 3498-3504.

[19] B. P. Williams, G. Klein, and I. Reid, "Automatic relocalization and loop closing for real-time monocular SLAM," IEEE Trans. Pattern Anal. Mach. Intell., vol. 33, no. 9, pp. 1699-1712, 2011. [Online]. Available: http://doi.ieeecomputersociety.org/10.1109/TPAMI.2011.41

[20] W. Tan, H. Liu, Z. Dong, G. Zhang, and H. Bao, "Robust monocular SLAM in dynamic environments," in IEEE International Symposium on Mixed and Augmented Reality, ISMAR 2013, Adelaide, Australia, October 1-4, 2013, 2013, pp. 209-218. [Online]. Available: http://dx.doi.org/10.1109/ISMAR.2013.6671781

[21] H. Johannsson, M. Kaess, M. F. Fallon, and J. J. Leonard, "Temporally scalable visual SLAM using a reduced pose graph," in 2013 IEEE International Conference on Robotics and Automation, Karlsruhe, Germany, May 6-10, 2013, 2013, pp. 54-61. [Online]. Available: http://dx.doi.org/10.1109/ICRA.2013.6630556

[22] B. Tordoff and D. W. Murray, "Guided sampling and consensus for motion estimation," in Computer Vision ECCV. Springer, 2002, pp. $82-96$.

[23] H. Strasdat, A. J. Davison, J. M. M. Montiel, and K. Konolige, "Double window optimisation for constant time visual SLAM," in IEEE International Conference on Computer Vision, ICCV 2011, Barcelona, Spain, November 6-13, 2011, 2011, pp. 2352-2359. [Online]. Available: http://dx.doi.org/10.1109/ICCV.2011.6126517

[24] Y. Li, N. Snavely, and D. P. Huttenlocher, "Location recognition using prioritized feature matching," in Computer Vision-ECCV 2010. Springer, 2010, pp. 791-804.

[25] R. Raguram, J. Tighe, and J.-M. Frahm, "Improved geometric verification for large scale landmark image collections." in BMVC, 2012, pp. $1-11$.

[26] G. H. Lee and M. Pollefeys, "Unsupervised learning of threshold for geometric verification in visual-based loop-closure," in Robotics and Automation (ICRA), 2014 IEEE International Conference on. IEEE, 2014, pp. 1510-1516.

[27] T. Kanji, "Cross-season place recognition using nbnn scene descriptor," in Intelligent Robots and Systems, 2015 IEEE/RSJ International Conference on. IEEE, 2015.

[28] — , "Unsupervised part-based scene modeling for visual robot localization," in ICRA. IEEE, 2015, pp. 6359-6365.

[29] A. Masatoshi, C. Yuuto, T. Kanji, and Y. Kentaro, "Leveraging imagebased prior in cross-season place recognition," in ICRA. IEEE, 2015, pp. 5455-5461.

[30] L. Enfu and T. Kanji, "Discriminative map matching using view dependent map descriptor," in IROS15 WS PPNIV, 2015.

[31] G. Grisetti, R. Kümmerle, C. Stachniss, and W. Burgard, "A tutorial on graph-based slam," Intelligent Transportation Systems Magazine, IEEE, vol. 2, no. 4, pp. 31-43, 2010.

[32] M. Brubaker, A. Geiger, R. Urtasun, et al., "Lost! leveraging the crowd for probabilistic visual self-localization," in Computer Vision and Pattern Recognition (CVPR), 2013 IEEE Conference on. IEEE, 2013, pp. 3057-3064. 